\title{
BMJ Open Acupuncture for patients with vascular dementia: a systematic review protocol
}

\author{
Yang Ye, ${ }^{1,2}$ Ling-Yong Xiao, ${ }^{1,2}$ Yi-Hua Liu, ${ }^{1}$ Jing-Wen Yang, ${ }^{2}$ Chao-Qun Yan, ${ }^{2}$ \\ Xue-Rui Wang, ${ }^{2}$ Guang-Xia Shi, ${ }^{2}$ Cun-Zhi Liü ${ }^{2}$
}

To cite: Ye Y, Xiao L-Y, Liu Y-H, et al. Acupuncture for patients with vascular dementia: a systematic review protocol. BMJ Open 2017;7:e019066. doi:10.1136/ bmjopen-2017-019066

- Prepublication history for this paper is available online To view these files, please visit the journal online(http://dx.doi. org/10.1136/bmjopen-2017019066).

YY and L-YX contributed equally.

Received 9 August 2017

Revised 19 September 2017

Accepted 26 September 2017

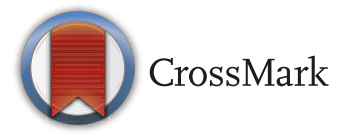

${ }^{1}$ Graduate School, Beijing University of Chinese Medicine, Beijing, China

${ }^{2}$ Department of Acupuncture and Moxibustion, Beijing Hospital of Traditional Chinese Medicine Affiliated to Capital Medical University, Beijing Key Laboratory of Acupuncture Neuromodulation, Beijing, China

Correspondence to

Professor Cun-Zhi Liu;

Icz623780@126.com

\begin{abstract}
Introduction This systematic review protocol aims to provide the methods used to evaluate the effectiveness and safety of acupuncture therapy for treating vascular dementia.

Methods and analysis The following eight databases will be searched from inception to July 2017: Cochrane Central Register of Controlled Trials, PubMed, MEDLINE, EMBASE, China National Knowledge Infrastructure, Chinese Biomedical Literature Database, VIP Database and Wanfang Database. All randomised controlled trials in English or Chinese related to acupuncture for vascular dementia will be included. Outcomes will include change in cognitive function and activities of daily living. The incidence of adverse events will be assessed for safety evaluation. Study inclusion, data extraction and quality assessment will be performed independently by two reviewers. Assessment of risk of bias and data synthesis will be performed using Review Manager software. Ethics and dissemination Ethics approval is not required because individual patient data are not included. The findings of this systematic review will be disseminated through peer-reviewed publication or conference presentations.
\end{abstract}

PROSPERO registration number CRD42017071820.

\section{INTRODUCTION}

\section{Description of the condition}

Vascular dementia (VaD) is the second most common form of dementia after Alzheimer's disease in elderly people. ${ }^{1}$ This disease is caused by brain damage from impaired blood flow to your brain over a long period. ${ }^{2}$ According to recent epidemiological survey, the prevalence of $\mathrm{VaD}$ among elderly Chinese individuals aged over 65 years was $1.5 \% .^{3}$ Furthermore, the risk of developing this form of dementia increases dramatically with age. ${ }^{4}$ Definitive treatment for $\mathrm{VaD}$ is still absent at present. ${ }^{5}$ The aim of current treatment is to control symptoms and correct the risk factors, but the effect is not satisfying. ${ }^{2}$

\section{Description of the intervention}

Acupuncture is an ancient Chinese healing technique that treats disorders by inserting needles into the skin. Its effectiveness against many diseases has been verified by a
Strengths and limitations of this study

This systematic review will comprehensively assess the effectiveness and safety of acupuncture therapy for treating vascular dementia.

- The study screening, data extraction and quality assessment will be performed by two independent reviewers.

- Different types of acupuncture may cause considerable heterogeneity in this review. Highquality trials might be deficient to generate convincing conclusions.

number of high-quality clinical trials. ${ }^{6-8}$ In addition, acupuncture is generally considered safe when performed correctly. The use of acupuncture treatment for 43 diseases has been recommended by the WHO. ${ }^{9}$ As a non-pharmacological intervention, acupuncture is considered to be a preferable alternative to pharmacotherapy for treating relevant outcomes in dementia such as behavioural disturbances. ${ }^{10}$ Furthermore, an increasing number of clinical studies have shown therapeutic effects of acupuncture on patients with VaD. ${ }^{1112}$

\section{How the intervention might work}

The potential mechanisms of acupuncture on VaD have been summarised in our previous review. ${ }^{13}$ Multiple aspects of the pathological process of VaD such as oxidative stress, apoptosis and neuroinflammation may be improved by acupuncture. ${ }^{13}$ Our recent research also showed that cognitive deficits in $\mathrm{VaD}$ rat models were attenuated by acupuncture through activation of D1/D5 receptors. ${ }^{14}$

\section{Why it is important to perform this review}

Currently, there are no licensed treatments for $\mathrm{VaD} .{ }^{1}$ Acupuncture has been used for years to treat $\mathrm{VaD}$ in China. ${ }^{15} 16$ However, the effectiveness of acupuncture for $\mathrm{VaD}$ remains controversial. There are so far two published systematic reviews referring to 'acupuncture' and 'vascular dementia'. ${ }^{5} 17$ A Cochrane systematic review published in 
2007 and updated in 2011 aimed to assess the efficacy and adverse effects of acupuncture for treating VaD. ${ }^{5}$ However, this review did not include any clinical trials because the criteria for including studies were quite strict. Only randomised controlled trials (RCTs) of acupuncture versus placebo or no intervention were included. Therefore, this systematic review almost did not reach any valuable conclusions, and 6years have passed since this review was updated in 2011. Many new trials in this field have been published within the past 6 years. ${ }^{11} 1819$ Another review published in 2017 evaluated the quality of reports about RCTs of scalp acupuncture for the treatment of VaD. ${ }^{17}$ Quality of included reports was the main aim to assess in this article, and scalp acupuncture is just one form of acupuncture treatment. To sum up, it remains unknown whether acupuncture is effective and safe for treating patients with VaD. Thus, it is important to perform a systematic review to obtain a relatively convincing conclusion whether acupuncture is a good choice to patients with $\mathrm{VaD}$.

\section{Objectives}

This systematic review aims to evaluate the effectiveness and safety of acupuncture therapy for patients with VaD. To this end, the following comparisons will be addressed:

1. Acupuncture versus sham acupuncture, placebo or no treatment.

2. Acupuncture versus western medicine, usual care or other therapies.

3. Acupuncture plus another treatment versus the same other treatment alone.

\section{METHODS}

Criteria for including studies in this review

Types of studies

RCTs of acupuncture therapy for VaD without any language or publication status restrictions will be included. Non-RCTs and uncontrolled clinical trials will be excluded.

\section{Types of participants}

Participants with a diagnosis of VD will be included regardless of their age, gender, race, education or economic status. The diagnosis will be based on the Hachinski Ischaemic Scale, Mini-Mental State Examination (MMSE), Hasegawa Dementia Scale (HDS), National Institute of Neurological Disorders and Stroke-Association Internationale pour la Recherche et l'Enseignement en Neurosciences criteria and the fourth edition of Diagnostic and Statistical Manual of Mental Disorders.

\section{Types of interventions}

Acupuncture therapy with needle insertion will be included, including body acupuncture, auricular acupuncture, scalp acupuncture, fire needling, warm needling and electroacupuncture. Point injection, laser acupuncture, moxibustion and cupping will be excluded. No restrictions on the treatment length or frequency.
Control interventions, including sham acupuncture (non-acupoint, minimal), placebo control, no treatment, western medicine, usual care and other conventional therapies, will be included. Trials evaluating acupuncture plus another treatment compared with the same other treatment alone will also be included. Studies comparing different acupoints or different forms of acupuncture will be excluded.

\section{Types of outcome measures}

The primary outcome measurements will be improvement in cognitive function and behavioural disturbances. ${ }^{20}$ The cognitive function will be measured by validated measurement scales such as MMSE, HDS, Blesse Dementia Scale and Montreal Cognitive Assessment. The behavioural disturbances will be assessed using validated scales such as Bless Behavior Scale.

The secondary outcome measures will include:

1. Activities of daily living: measured by Activity of Daily Living Scale and Functional Activities Questionnaire.

2. Safety: measured by incidence and severity of adverse effects.

3. Acceptability of treatment as measured by withdrawal from trials.

\section{Search methods for identification of studies Electronics searches}

The following electronic databases will be searched from inception to July 2017 regardless of language and publication status: Cochrane Central Register of Controlled Trials, PubMed, MEDLINE, EMBASE, China National Knowledge Infrastructure, Chinese Biomedical Literature Database, VIP Database and Wanfang Database.

The following terms will be searched: $\mathrm{VD}$, VaD, vascular dementia, acupuncture, body acupuncture, scalp acupuncture, auricular acupuncture, fire needling, warm needling and electroacupuncture. The search strategy for PubMed is shown in table 1 . The equivalent search words will be used in the Chinese databases.

\section{Searching other resources}

Ambiguous literature will be investigated manually to avoid missing eligible trials. Reference lists of identified publications will also be manually searched. In addition, the following journals published in Chinese will be searched as a supplement: Acupuncture Research (1976July 2017), Chinese Acupuncture and Moxibustion (1981July 2017), Journal of Clinical Acupuncture and Moxibustion (1985-July 2017), Journal of Traditional Chinese Medicine (1960-July 2017) and Shanghai Journal of Acupuncture and Moxibustion (1982-July 2017).

\section{Data collection and analysis}

Selection of studies

The titles and abstracts of all searched studies will be identified by two independent reviewers (YY and Y-HL) according to the inclusion criteria. The full text will be reviewed if necessary. Any disagreements will be resolved through discussion with a third reviewer (X-RW). 


\begin{tabular}{|c|c|}
\hline Number & Search items \\
\hline 1 & randomised controlled trial.pt \\
\hline 2 & controlled clinical trial.pt \\
\hline 3 & randomised.ti,ab \\
\hline 4 & randomised.ti,ab \\
\hline 5 & randomly.ti,ab \\
\hline 6 & placebo.ti,ab \\
\hline 7 & trial.ti,ab \\
\hline 8 & 1 or $2-7$ \\
\hline 9 & dementia.ti,ab \\
\hline 10 & vascular dementia.ti,ab \\
\hline 11 & cognitive disorders.ti,ab \\
\hline 12 & cognitive impairment.ti,ab \\
\hline 13 & 9 or $10-12$ \\
\hline 14 & acupuncture.ti,ab \\
\hline 15 & acupuncture therapy.ti,ab \\
\hline 16 & acupoints.ti,ab \\
\hline 17 & acupuncture points.ti,ab \\
\hline 18 & body acupuncture.ti,ab \\
\hline 19 & scalp acupuncture.ti,ab \\
\hline 20 & auricular acupuncture.ti,ab \\
\hline 21 & ear acupuncture.ti,ab \\
\hline 22 & manual acupuncture.ti,ab \\
\hline 23 & electroacupuncture.ti,ab \\
\hline 24 & electro-acupuncture.ti,ab \\
\hline 25 & fire needling.ti,ab \\
\hline 26 & warm needling.ti,ab \\
\hline 27 & 14 or $15-26$ \\
\hline 28 & 8 and 13 and 27 \\
\hline
\end{tabular}

Excluded studies will be listed in a table with reasons for their exclusion. The study selection procedure is shown in figure 1 .

\section{Data extraction and management}

A standard data extraction form will be created before data extraction. The data extraction form will include author information, year of publication, participants, randomisation, inclusion and exclusion criteria, acupuncture intervention, control intervention, outcomes and adverse events. All the study data will be extracted by two independent reviewers (YY and Y-HL). Any disagreements will be discussed and finally judged by a third reviewer (X-RW). Only the latest report will be included when a same trial was described by multiple publications. Data not available in the publications will be obtained by contacting corresponding authors for more information. All data will be cross-checked by YY and Y-HL and transferred into Review Manager software.
Assessment of risk of bias

The risk and bias in included studies will be assessed by two independent reviewers (LY and G-XS) using the Cochrane Collaboration's tool. ${ }^{19}$ The following domains will be evaluated: selection bias, performance bias, detection bias, attrition bias, reporting bias and other sources of bias. The assessments will then be classified into three levels: low risk, high risk and unclear. Unclear items in studies will be inquired by contacting corresponding authors for details. Any disagreement will be resolved by discussion with a third reviewer (X-RW).

\section{Measures of treatment effect}

For dichotomous data, risk ratio with $95 \%$ CIs will be used for analysis. For continuous data, mean difference with $95 \%$ CIs will be used for analysis. Standardised mean difference with $95 \%$ CIs will be used if different scales were used to measure a certain outcome variable.

\section{Unit of analysis issues}

The unit of analysis will be the individual participant.

\section{Dealing with missing data}

The corresponding authors will be contacted by reviewers (YY and Y-HL) to obtain missing data. If the missing data are unobtainable, an intention-to-treat analysis will be performed if possible and a sensitivity analysis will be conducted to address the potential impact of missing data. ${ }^{21} 22$ The impact of missing data will be discussed if necessary.

\section{Assessment of heterogeneity}

A standard $\chi^{2}$ test with a significance level of $\mathrm{p}<0.1$ will be used for testing statistical heterogeneity. An $\mathrm{I}^{2}$ test will be used for quantifying inconsistency among the included studies. Study will not be considered to have heterogeneity when the $\mathrm{I}^{2}$ value is less than $50 \%$.

\section{Assessment of reporting biases}

Funnel plots will be used to detect the potential reporting biases if more than 10 studies are included. The Egger's test will be used to determine funnel plot asymmetry.

\section{Data synthesis}

Data synthesis will be performed using Review Manager software provided by Cochrane Collaboration. The fixed-effects model will be used for pooled data if no substantial statistical heterogeneity is detected. The random-effects model will be used to combine the data if there is substantial statistical heterogeneity. Subgroup analysis will be performed or the potential reasons will be analysed if significant heterogeneity between studies is found.

\section{Subgroup analysis and investigation of heterogeneity}

Subgroup analysis will be performed to interpret the heterogeneity if possible. Factors like different acupuncture types and different control interventions will be taken into account. 


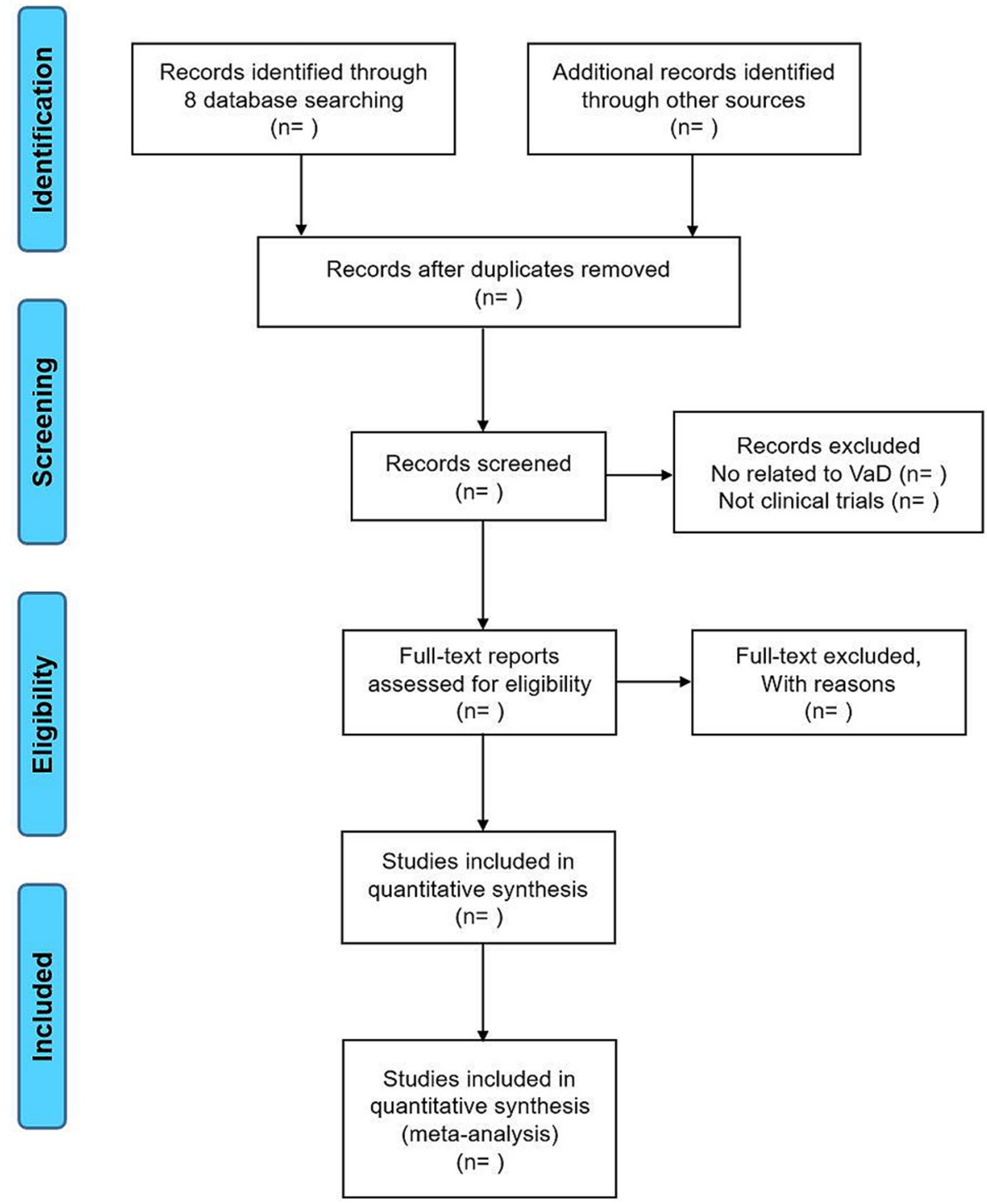

Figure 1 Flow diagram of the study selection process. VaD, vascular dementia.

\section{Sensitivity analysis}

A sensitivity analysis will be conducted to verify the robustness of the review conclusions, if possible. The impacts of methodological quality, sample size and missing data will be evaluated. Additionally, the analysis will be repeated after low-methodological-quality studies are excluded.

\section{Summary of evidence}

Results of the outcomes will be summarised in 'Summary of findings' tables. The quality of evidence for all outcomes will be assessed through the Grading of Recommendations Assessment Development and Evaluation approach. ${ }^{19}$ The assessments will be adjudicated into four levels: high, moderate, low and very low quality.

\section{Ethics and dissemination}

Ethics approval will not be needed because date from individual patients will not be included and no privacy will be involved. The results of this systematic review will be disseminated through peer-reviewed publications or conference presentations. The essential protocol amendments will be documented in the full review.

\section{DISCUSSION}

This systematic review will provide an assessment of the current state of acupuncture treatment for VaD. Conclusions drawn from this review may benefit patients with VaD, clinicians and policy makers. The process of conducting this review will be divided into four parts: identification, study inclusion, data extraction and data synthesis. This review has some potential limitations. First, various forms of acupuncture may cause considerable heterogeneity. Second, the quality of included reports might be poor, which will limit the ability to generate conclusions based on high confidence.

Contributors $\mathrm{YY}$ and C-ZL designed the systematic review. YY drafted the protocol and $L-Y X, Y-H L, J-W Y$ and $C-Q Y$ revised the manuscript. YY and $Y-H L$ will independently screen the potential studies, extract data, assess the risk of bias and 
finish data synthesis. X-RW and G-XS will arbitrate any disagreements during the review. All authors approved the publication of the protocol.

Funding This work was funded by the National Natural Science Foundation of China (grant numbers 81473501 and 81303122).

Competing interests None declared.

Patient consent Detail has been removed from this case description/these case descriptions to ensure anonymity. The editors and reviewers have seen the detailed information available and are satisfied that the information backs up the case the authors are making.

Provenance and peer review Not commissioned; externally peer reviewed.

Open Access This is an Open Access article distributed in accordance with the Creative Commons Attribution Non Commercial (CC BY-NC 4.0) license, which permits others to distribute, remix, adapt, build upon this work non-commercially, and license their derivative works on different terms, provided the original work is properly cited and the use is non-commercial. See: http://creativecommons.org/ licenses/by-nc/4.0/

(c) Article author(s) (or their employer(s) unless otherwise stated in the text of the article) 2017. All rights reserved. No commercial use is permitted unless otherwise expressly granted.

\section{REFERENCES}

1. O'Brien JT, Thomas A. Vascular dementia. Lancet 2015;386:1698-706.

2. Iadecola $\mathrm{C}$. The pathobiology of vascular dementia. Neuron 2013;80:844-66.

3. Jia J, Wang F, Wei C, et al. The prevalence of dementia in urban and rural areas of China. Alzheimers Dement 2014;10:1-9.

4. Song J, Lee WT, Park KA, et al. Association between risk factors for vascular dementia and adiponectin. Biomed Res Int 2014;2014:261672:1-13.

5. Peng WN, Zhao H, Liu ZS, et al. Acupuncture for vascular dementia Cochrane Database Syst Rev 2007:CD004987.

6. Liu Z, Liu Y, Xu H, et al. Effect of electroacupuncture on urinary leakage among women with stress urinary incontinence: a randomized clinical trial. JAMA 2017;317:2493-501.

7. Zhao L, Chen J, Li Y, et al. The long-term effect of acupuncture for migraine prophylaxis: a randomized clinical trial. JAMA Intern Med 2017;177:508-15.
8. Liu Z, Yan S, Wu J, et al. Acupuncture for chronic severe functional constipation: a randomized trial. Ann Intern Med 2016;165:761-9.

9. Zhao ZQ. Neural mechanism underlying acupuncture analgesia. Prog Neurobiol 2008;85:355-75.

10. Abraha I, Rimland JM, Trotta FM, et al. Systematic review of systematic reviews of non-pharmacological interventions to treat behavioural disturbances in older patients with dementia. The SENATOR-OnTop series. BMJ Open 2017;7:e012759.

11. Liu Q, Wang XJ, Zhang ZC, et al. Neuroprotection against vascular dementia after acupuncture combined with donepezil hydrochloride: P300 event related potential. Neural Regen Res 2016;11:460-4.

12. Shi GX, Liu CZ, Guan W, et al. Effects of acupuncture on Chinese medicine syndromes of vascular dementia. Chin J Integr Med 2014;20:661-6.

13. Ye $\mathrm{Y}$, Zhu W, Wang XR, et al. Mechanisms of acupuncture on vascular dementia-A review of animal studies. Neurochem Int 2017;107:204-10.

14. Ye Y, Li H, Yang JW, et al. Acupuncture attenuated vascular dementia-induced hippocampal long-term potentiation impairments via activation of D1/D5 receptors. Stroke 2017;48:1044-51.

15. Chen B. Clinical observation on acupuncture treatment of cerebrovascular dementia-a report of 32 cases. J Tradit Chin Med 2001;21:174-6.

16. Gao H, Yan L, Liu B, et al. Clinical study on treatment of senile vascular dementia by acupuncture. J Tradit Chin Med 2001;21:103-9.

17. You YN, Cho MR, Park JH, et al. Assessing the quality of reports about randomized controlled trials of scalp acupuncture treatment for vascular dementia. Trials 2017;18:205.

18. Li SK, Ding DM, Zhang ZL, et al. Effects of scalp acupuncture combined with auricular point sticking on cognitive behavior ability in patients with vascular dementia. Zhongguo Zhen Jiu 2014;34:417-20.

19. Wang $\mathrm{S}$, Yang $\mathrm{H}$, Zhang J, et al. Efficacy and safety assessment of acupuncture and nimodipine to treat mild cognitive impairment after cerebral infarction: a randomized controlled trial. BMC Complement Altern Med 2016;16:361.

20. Abraha I, Cruz-Jentoft A, Soiza RL, et al. Evidence of and recommendations for non-pharmacological interventions for common geriatric conditions: the SENATOR-ONTOP systematic review protocol. BMJ Open 2015;5:e007488.

21. Abraha I, Cozzolino F, Orso M, et al. A systematic review found that deviations from intention-to-treat are common in randomized trials and systematic reviews. J Clin Epidemiol 2017;84:37-46.

22. Porta N, Bonet $\mathrm{C}$, Cobo E. Discordance between reported intentionto-treat and per protocol analyses. J Clin Epidemiol 2007;60:663-9. 